\title{
Search for single top production in ep collisions at HERA
}

\section{Stefania Xella*}

University of Zurich

Winterthurerstrasse 190, $\mathrm{CH}-8057$ Zurich, $\mathrm{CH}$

E-mail: xella@physik.unizh.ch

\begin{abstract}
Results on the search for single production of top quarks at the $\mathrm{H} 1$ and ZEUS experiments at HERA are presented, based on the analysis of a data sample collected in the years from 1994 to 2000 (HERA I). Decays of top quarks into a b quark and a W boson are considered in the leptonic and hadronic decay channels of the W. In the absence of a clear signal for anomalous single top production, via Flavour Changing Neutral Currents, limits on the anomalous magnetic coupling constants $\mathrm{k}_{\text {tuy }}$ and $\mathrm{v}_{\text {tuz }}$ are set.

Additionally, some recent results from both experiments on the full 1994 to 2005 data sample (HERA I and HERA II) on the isolated leptons search, which constitutes the preselection step in the single top search in the leptonic channel, are shown.
\end{abstract}

International Workshop on Top Quark Physics

University of Coimbra, Portugal

12-15 January, 2006

\footnotetext{
${ }^{*}$ Speaker
} 


\section{Introduction}

According to the Standard Model (SM), the expected production cross section for single top quarks in electron or positron on proton collisions at HERA is about $1 \mathrm{fb}$. This means about 0.1 events expected in $100 \mathrm{pb}^{-1}$ of data, which is a negligible number. Therefore the observation of single top production at HERA would be a clear signal of new physics.

At HERA a sizeable production of single top quarks could be achieved via processes involving Flavour Changing Neutral Currents (FCNC), see fig 1. FCNC interactions are present in several extensions of the SM, like SuperSymmetry [1], and have been considered previously in the analysis of data at LEP [2] and TEVATRON [3]. At HERA one can set limits on the coupling of $u$ quark to top quark in FCNC interactions mediated by a $\gamma$ or a $\mathrm{Z} 0$ boson, the latter being suppressed because of the large mass of the Z0 boson. The sensitivity to the coupling of a top quark to a charm quark in such FCNC processes is suppressed at HERA because of the negligible c quark content of the proton.

In this article we describe the results of the search for single top production performed at HERA on the data collected between 1994 and 2000 (HERA I) by the two experiments, H1 and ZEUS [4,5]. In section 2 we describe the signatures of single top production and the main backgrounds to the search. In section 3 and 4 we describe the selection in details, adding also some more recent results on isolated lepton searches based on the full data sample collected in 1994-2005. In section 5 we present the final HERA I results. In section 6 we give some conclusions.

\section{Signatures of single top production}

At HERA the search for single top quarks concentrates on the decay $t \rightarrow b W$, with $\mathrm{W}$ decaying either into a lepton and a neutrino (leptonic channel) or into a couple of quarks (hadronic channel).

In the leptonic channel, the signatures of a single top decay are large missing momentum (due to the neutrino), an isolated lepton of high $\mathrm{p}_{\mathrm{T}}$ (originating from the $\mathrm{W}$ ), and a high $\mathrm{p}_{\mathrm{T}}$ hadronic system (originating from the $b$ quark decay products)

In the hadronic channel, the signature of a single top decay are three hadronic jets, two originating from the $\mathrm{W}$ decay and one coming from the $\mathrm{b}$ quark.

These signatures are exploited when implementing the selection described in the next sections. 
Backgrounds to the search for single top production are different for the two $\mathrm{W}$ decay channels. In the leptonic channel the main background comes from $\mathrm{W}$ production, which is sizeable and has similar features to single top events: a W and a hadronic system, though the latter tends to have a smaller $\mathrm{p}_{\mathrm{T}}$ than the one originating from $\mathrm{a} b$ quark present in top decays.

Additional backgrounds in the leptonic channel are coming from Neutral Current (NC), Charged Current (CC) and photoproduction events, where either there is a genuine isolated lepton and a large missing $\mathrm{p}_{\mathrm{T}}$ due to mismeasurements ( $\mathrm{NC}$ and photoproduction), or there is genuine large missing $\mathrm{p}_{\mathrm{T}}$ from a neutrino and a misidentified lepton (CC).

Backgrounds to the hadronic channel mainly come from three jet events in photoproduction and NC.

\section{Search for single top in the leptonic channel}

\subsection{Preselection: Search for events with an isolated lepton and large missing $p_{T}$}

The search for single top in the leptonic channel starts off as a search for events with an isolated lepton and large missing total $\mathrm{p}_{\mathrm{T}}$ (the signatures of $\mathrm{W}$ decay). As shown in table 1, H1 and ZEUS apply a very similar preselection. $\mathrm{D}_{\text {jet }}, \mathrm{D}_{\text {track }}$ are the angular distances of the isolated lepton from other jets and tracks respectively, and by acoplanarity it is meant the azimuthal angular distance of the isolated lepton to the hadronic system X. Additionally, ZEUS requires the presence of one jet in the event, with large pT.

\begin{tabular}{|l|l|l|}
\hline & $\mathrm{H} 1$ & ZEUS \\
\hline Lepton within detector & $5<\theta<140$ degrees & $\theta<115$ degrees \\
\hline High $\mathrm{p}_{\mathrm{T}}$ of lepton & $\mathrm{p}_{\mathrm{T}}>10 \mathrm{GeV}$ & $\mathrm{p}_{\mathrm{T}}>5 \mathrm{GeV}$ \\
\hline Lepton Isolation & $\mathrm{D}_{\text {track }}>0.5 \mathrm{rad}$ & $\mathrm{D}_{\text {track }}>0.5 \mathrm{rad}$ \\
& $\mathrm{D}_{\text {jet }}>1.0 \mathrm{rad}$ & $\mathrm{D}_{\text {jet }}>1.0 \mathrm{rad}$ \\
\hline Large Missing total $\mathrm{pT}$ & $\mathrm{p}_{\mathrm{T}}{ }^{\text {miss }}>12 \mathrm{GeV}$ & $\mathrm{p}_{\mathrm{T}}{ }^{\text {miss }}>20 \mathrm{GeV}$ \\
\hline Acoplanarity & e: $\phi$ acopl $<160$ degrees & $\phi$ acopl $<172$ degrees \\
& $\mu: \phi$ acopl $<170$ degrees & \\
\hline
\end{tabular}

Table 1: Isolated lepton preselection, in the search for single top quarks

The results of the search for events with an isolated lepton and large $\mathrm{p}_{\mathrm{T}}{ }^{\text {miss }}$ in $\mathrm{H} 1$ and ZEUS are summarized in table 2 and described in detail in [5,6]. While on the full sample of data there is a general good agreement between data and expectation from MC simulation for both experiments, in the region of large $\mathrm{p}_{\mathrm{T}}$ for the hadronic system, denoted by $\mathrm{X}, \mathrm{H} 1$ observes an excess of data with respect to the total SM expectation, which is not confirmed by the ZEUS measurements in that region. This excess is observed already when analyzing the data from the period 1994-2000 (HERA I, used in the search for single top presented here and in [4,5]) and is clear also in the full data set collected by H1 in the years 1994-2005, as can be seen in the distribution for $\mathrm{p}_{\mathrm{T}}{ }^{\mathrm{x}}$ in $\mathrm{H} 1$ data shown in figure 2. The excess is larger in the positron-proton 
collisions sample alone (3.4 standard deviations), than in the full sample (about 2 standard deviations), due to the fact the no excess is observed in electron-proton collisions.
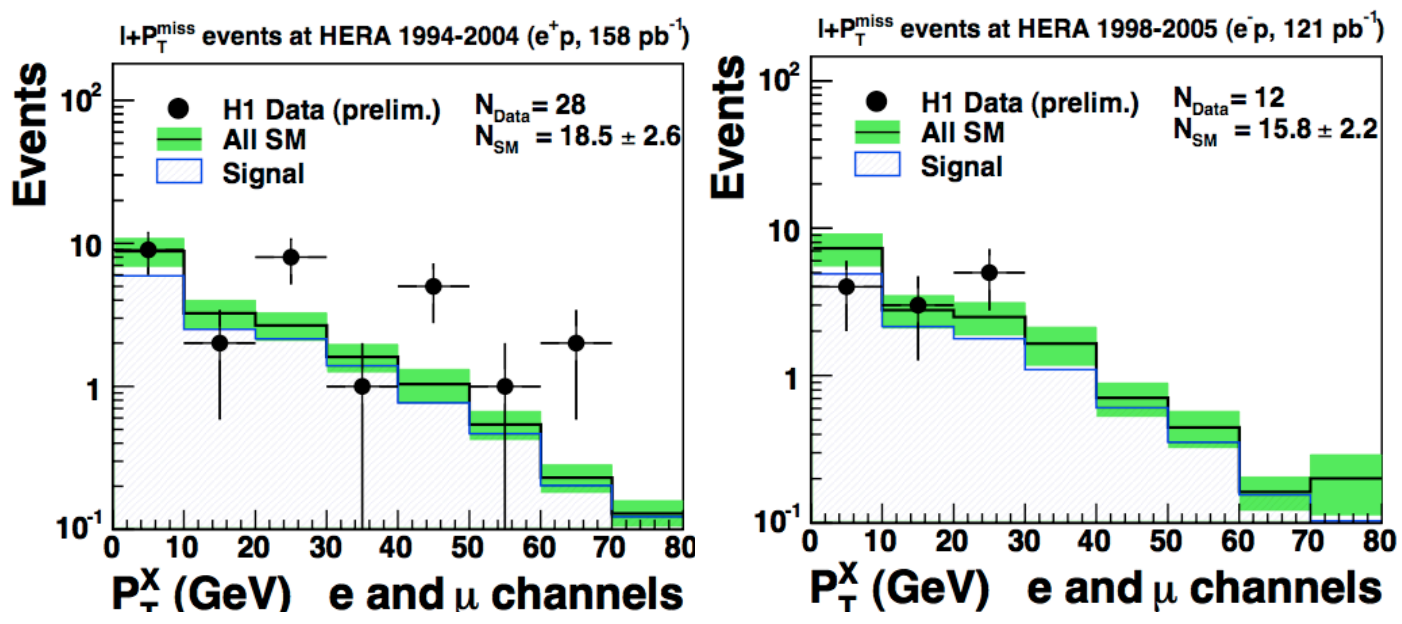

Figure 2: Distribution of the transverse momentum of the hadronic system in H1 data, in the search for events with isolated leptons and large missing $\mathrm{pT}$. The luminosity corresponds to the full 1994-2005 data sample.

\begin{tabular}{|c|c|c|c|c|}
\hline \multirow{3}{*}{$\begin{array}{l}\text { H1 } \\
1994-2005\end{array}$} & & Combined e/ $\mu$ & esults obs./exp. & SM W contrib. \\
\hline & \multirow{2}{*}{$\begin{array}{l}\mathrm{e}^{ \pm} \mathrm{p} \\
279 \mathrm{pb}^{-1}\end{array}$} & Full sample & $40 / 34.3 \pm 4.8$ & $71 \%$ \\
\hline & & $\mathrm{p}_{\mathrm{T}}^{\mathrm{X}}>25 \mathrm{GeV}$ & $17 / 9.0 \pm 1.5$ & $73 \%$ \\
\hline \multirow{2}{*}{$\begin{array}{l}\text { H1 (HERA I) } \\
1994-2000\end{array}$} & \multirow{2}{*}{$\begin{array}{l}\mathrm{e}^{ \pm} \mathrm{p} \\
118.3 \mathrm{pb}^{-1}\end{array}$} & Full sample & $18 / 12.4 \pm 1.7$ & $75 \%$ \\
\hline & & $\mathrm{P}_{\mathrm{T}}^{\mathrm{X}}>25 \mathrm{GeV}$ & $10 / 2.9 \pm 0.5$ & $87 \%$ \\
\hline \multirow{2}{*}{$\begin{array}{l}\text { ZEUS (HERA I) } \\
1994-2000\end{array}$} & \multirow{2}{*}{$\begin{array}{l}\mathrm{e}^{ \pm} \mathrm{p} \\
130.1 \mathrm{pb}^{-1}\end{array}$} & Full sample & $36 / 32.5 \pm 2.3$ & $17 \%$ \\
\hline & & $\mathrm{P}_{\mathrm{T}}^{\mathrm{X}}>25 \mathrm{GeV}$ & $7 / 5.6 \pm 1.4$ & $50 \%$ \\
\hline
\end{tabular}

Table 2: Results on the search for events with an isolated lepton and large missing $\mathrm{p}_{\mathrm{T}}$ in H1 and ZEUS in HERA I data. The most recent results from H1 on the full 1994-2005 data set are presented too. The contribution in \% of SM W to the total SM expectation is indicated.

More recent results from ZEUS [7], including data collected up to 2004 for the electron channel only and a selection procedure which yields a background expectation similar to the H1 analysis, give 1 event obs. / $1.5 \pm 0.18$ expected (with 78\% contribution from SM W production) at $\mathrm{p}_{\mathrm{T}}{ }^{\mathrm{x}}>25 \mathrm{GeV}$.

Top decays $\mathrm{t} \rightarrow \mathrm{b} \mathrm{W}$, with subsequent decay of $\mathrm{W}$ into lepton and neutrino, tend to have a large $\mathrm{p}_{\mathrm{T}}{ }^{\mathrm{x}}$, due to the presence of an energetic $\mathrm{b}$ quark jet with large $\mathrm{p}_{\mathrm{T}}$ originating from the top 
quark. This is why the excess observed in the $\mathrm{H} 1$ analysis at the preselection level propagates then to the final stage of the selection for single top quark production.

More recently there have been results also on isolated tau leptons for both experiments [8], but they are not used in the search for single top here presented.

\subsection{Final selection}

In the final selection of single top candidates in the HERA I data sample, more specific requirements are imposed, to constrain the kinematic and characteristics of the event to that one of a top decay. H1 requires the neutrino kinematics to be reconstructed, and the charge of the lepton to be positive (since mainly top and not anti-top is expected to be produced in electron proton collisions). Then both experiments use addditional observables like the $\mathrm{p}_{\mathrm{T}}$ of the $\mathrm{b}$ quark jet, the reconstructed mass of the top, and the $\mathrm{W}$ decay angle ${ }^{*}$, to finalize the search. They either apply cuts on these variables (ZEUS, H1) or combine these variables in a multivariate likelihood method (H1) and apply a cut on a discriminator $D$, defined as the probability for the event to be more background like ( $D$ values close to 0 ) or more top like ( $D$ values close to 1 ). For details of the final selection we refer the reader to $[4,5]$. The final result of the search in the leptonic channel are shown in table 3.

The distribution of the discriminator $D$ in the $\mathrm{H} 1$ analysis is shown in figure 3 , with the expected contribution from FCNC top production in a dashed red line. The 5 events selected in the leptonic channel are higly concentrated in the region where anomalous top production contributes.

\section{Search for single top in the hadronic channel}

\subsection{Preselection}

A top quark decaying in a $\mathrm{b}$ quark and a $\mathrm{W}$, with the $\mathrm{W}$ decaying hadronically, typically deposits a large amount of transverse energy in the detector, so a large total hadronic transverse energy and the presence of at least three high $\mathrm{p}_{\mathrm{T}}$ jets are required. Further, a pair of these jets should have an invariant mass compatible within two sigma with the $\mathrm{W}$ mass. At this level of preselection both experiments show a good agreement of data and MC expectation [4,5].

\subsection{Final selection}

The observables used for the discrimination of the top signal from the QCD background are chosen in analogy to the leptonic channel (section 3.2). The jet which was not used to form the $\mathrm{W}$ mass in the preselection is assigned to be the $\mathrm{b}$ quark jet, and then the same variables

\footnotetext{
* defined as the angle between the charged lepton momentum in the $\mathrm{W}$ rest frame and the $\mathrm{W}$ direction in the top quark rest frame
} 
described in sect. 3.2 are constructed, with the $\mathrm{W}$ decay angle now defined by the jet which has the lowest $\mathrm{p}_{\mathrm{T}}$ among the two assigned to the $\mathrm{W}$.

Both a selection based on cuts on these variables (ZEUS, H1) and on a discriminator (H1) are performed, as described in [4,5]. In figure 3 the distribution of the discriminator $D$ is shown, and the results for the selection in this channel are shown in table 3.
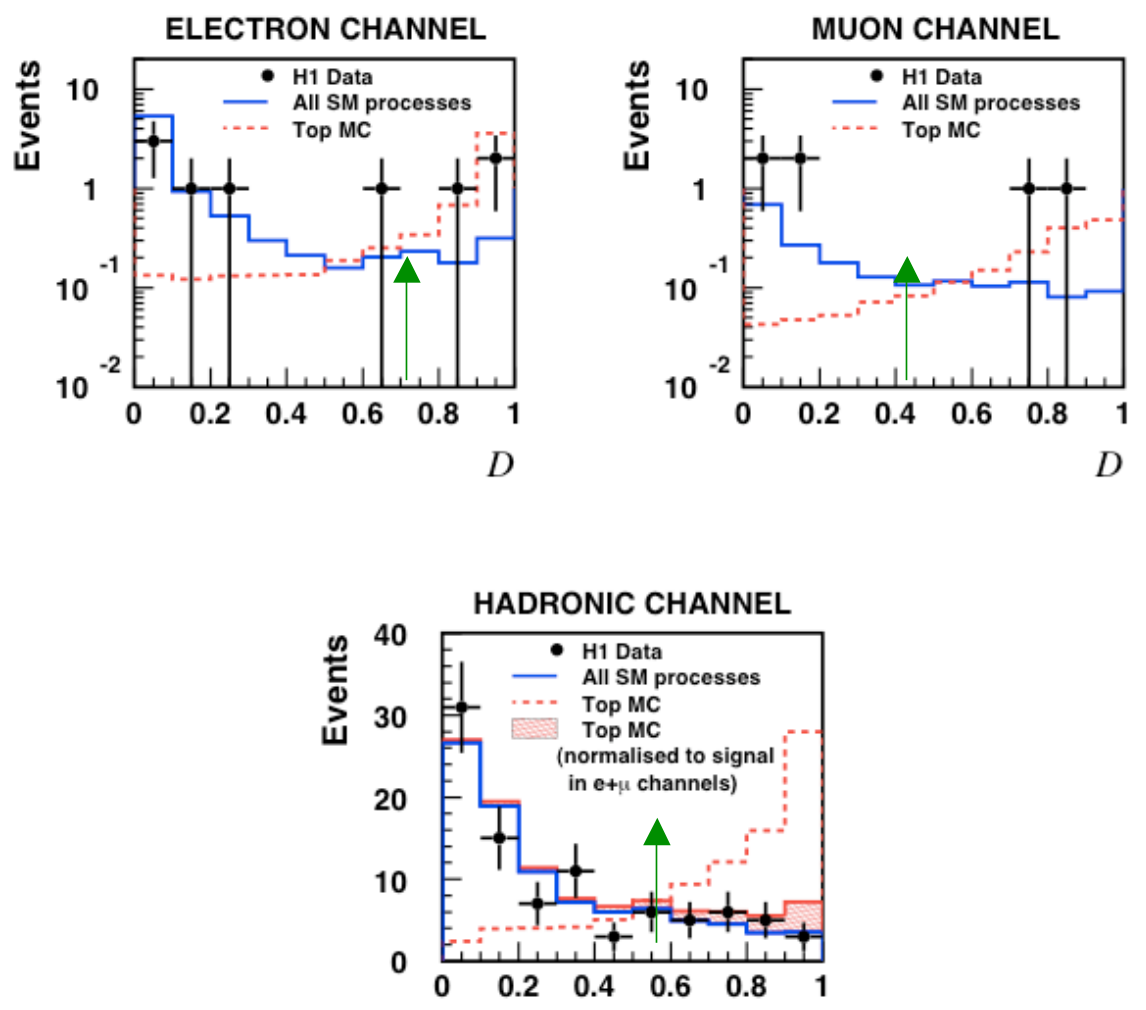

Figure 3: Distribution of the discriminator $D$ used for the final selection of single top quark production in $\mathrm{H} 1$. The contribution from FCNC anomalous top production is shown with a dashed red line. The final cut value for $D$ is also indicated in the figure.

\section{Results on single top search at HERA}

The H1 experiment observes a slight excess in the number of observed single top candidates in the leptonic channel with respect to the SM background expectation (see table 2, $3)$. Based on this result, $\mathrm{H} 1$ provides a measurement for single top production cross section in this channel $\sigma=0.41^{+0.29}-0.19 \mathrm{pb}$ at $\sqrt{\mathrm{s}}=319 \mathrm{GeV}$ which is more than two standard deviations away from zero. For the hadronic channel a cross section of $0.04^{+0.27}-0.23$ pb at $\sqrt{s}_{\mathrm{s}}=319 \mathrm{GeV}^{\dagger}$ is measured, consistent with no top signal observed. The two results are compatible within 1.1 sigma, and a combination of the two results gives a cross section of $\sigma=0.29^{+0.15}-0.14 \mathrm{pb}$ at $\sqrt{\mathrm{s}}=319 \mathrm{GeV}$ for single top production measured by $\mathrm{H} 1$.

\footnotetext{
${ }^{\dagger}$ using the results from the multivariate method
} 
Additionally, in view of the small number of single top candidates, and assuming the coupling of top to $\mathrm{u}$ quark via $\mathrm{Z} 0, \mathrm{v}_{\mathrm{tuZ}}$, to be zero, an upper limit on single top production cross section is calculated at the $95 \%$ C.L. at $\sqrt{s}_{\mathrm{s}}=319 \mathrm{GeV}$ by the $\mathrm{H} 1$ experiment. This result is shown in table 4 , together with the upper limit in $\mathrm{k}_{\text {tur }}$.

From the results shown in table 3 it is clear that in the case of the ZEUS experiment the number of observed candidates for single top production is compatible with the predicted SM background hence no evidence for single top production is seen. As a result of this only an upper limit at the $95 \%$ C.L. can be set on the cross section for single top production and on the anomalous FCNC coupling of $\mathrm{u}$ quark to top quark. This is shown in table 4 (where $\mathrm{v}_{\text {tuz }}$ is set to 0 ) and in figure 4 (where $v_{\text {tuz }}$ is left free). As one can see the limits are more stringent than those set by other experiments at LEP [2] and TEVATRON [3], and by the H1 experiment.

\begin{tabular}{|l|c|l|c|}
\hline channel & H1 cut based (eff.) & ZEUS cut based (eff.) & H1 multivariate (eff.) \\
\hline lepton & $5 / 1.31 \pm 0.22(37 \%)$ & $0 / 1.89 \pm 0.22(7 \%)$ & $5 / 1.29 \pm 0.25(37 \%)$ \\
\hline hadron & $18 / 20.2 \pm 3.6(30 \%)$ & $14 / 17.6 \pm 2 .(16.5 \%)$ & $20 / 17.5 \pm 3.2(30 \%)$ \\
\hline
\end{tabular}

Table 3 : Results of the search for single top quark production in $\mathrm{H} 1$ and ZEUS (efficiency of selection on simulated FCNC top signal quoted in parenthesis). The results correspond to the 1994-2000 (HERA I) data taking period, and a luminosity respectively of 118.3 and $130.1 \mathrm{pb}^{-1}$

\begin{tabular}{|c|c|l|c|c|}
\hline & Lumi & \multicolumn{1}{|c|}{ channel } & $\sigma(\mathrm{pb})$ & $\mathrm{k}_{\text {tuy }}$ \\
\hline $\mathrm{H} 1$ & $\mathrm{e}^{ \pm} \mathrm{p}$ & lepton & $<0.90$ & $<0.35$ \\
\cline { 3 - 5 } $1994-2000$ & $118.3 \mathrm{pb}^{-1}$ & hadron & $<0.48$ & $<0.25$ \\
\cline { 3 - 5 } & & combined & $<0.55$ & $<0.27$ \\
\hline ZEUS & $\mathrm{e}^{ \pm} \mathrm{p}$ & lepton & $<0.91 / 0.51$ & $<0.22$ \\
\cline { 3 - 5 } 1994-2000 & \multirow{2}{*}{$130.1 \mathrm{pb}^{-1}$} & hadron & $<0.99 / 0.43$ & $<0.24$ \\
\cline { 3 - 5 } & & combined & $<0.22$ & $<0.17$ \\
\hline
\end{tabular}

Table 4: Exclusion limits at the 95\% confidence level for the single top cross section and the anomalous tur coupling, based on the analysis of HERA I data. Results of H1 are at $\sqrt{\mathrm{s}}=319 \mathrm{GeV}$, while for ZEUS the results are quoted at $\sqrt{\mathrm{s}}=318 \mathrm{GeV}$ for the combined result, and at $\sqrt{\mathrm{s}}=300 / 318 \mathrm{GeV}$ for the single channel results

\section{Conclusions}

The H1 and ZEUS experiments at HERA have studied the anomalous production of top quarks in FCNC models in HERA I data (1994-2000). The H1 experiment observes a slight 
excess of data over the SM prediction, and based on this quotes a measurement of cross section for anomalous single top production which is more than two standard deviations away from zero. Alternatively, due to the low number of top quark candidates observed, the H1 experiment, and similarly the ZEUS experiment, can set an upper limit on the cross section for single top production and on anomalous top coupling to u quarks. The ZEUS limits are more stringent than the limits the H1 experiment and experiments at LEP and the TEVATRON are able to set.

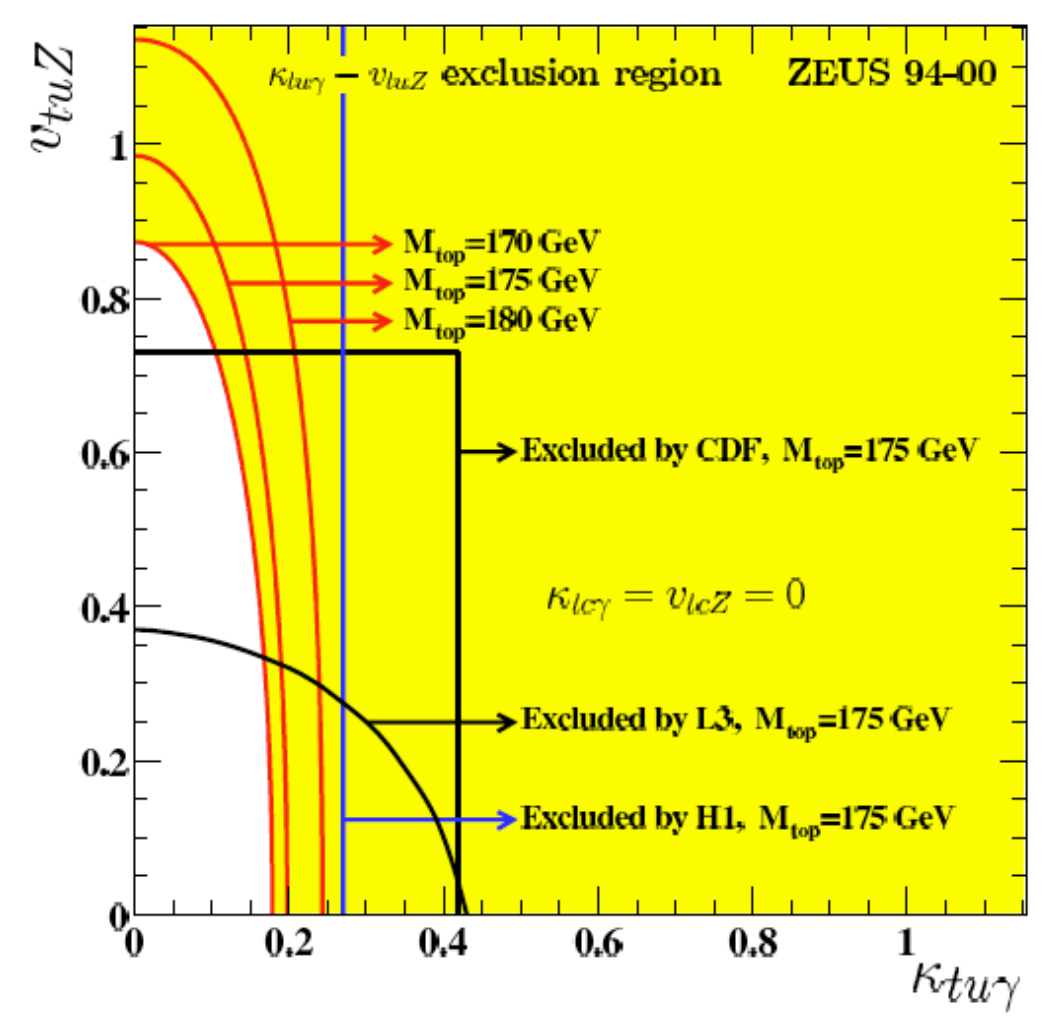

Figure 4: ZEUS exclusion regions at 95\% C.L. in the $\mathrm{k}_{\mathrm{tu}_{\gamma}}-\mathrm{v}_{\mathrm{tuZ}}$ plane for three values of the $\mathrm{M}_{\text {top }}$ assuming $\mathrm{k}_{\mathrm{tcy}}=\mathrm{v}_{\mathrm{tcZ}}=0$. The CDF, L3 and H1 exclsuion limits for $\mathrm{M}_{\text {top }}=$ $175 \mathrm{GeV}$ are also shown.

\section{References}

[1] G.M. de Divitiis, R. Petronzio, L.Silvestrini, Nucl.Phys.B 504 (1997) 45 [hep-ph / 9704244 ]

[2] P.Achard et al., L3 collaboration, Phys.Lett.B 549 (2002) 290 [hep-ex/ 0210041 ] , A.Heister et al., ALEPH collaboration, Phys.Lett.B 543 (2002) 173 [hep-ex/ 0206070 ], G.Abbiendi et al., OPAL collaboration, Phys.Lett.B 521 (2001) 181 [hep-ex/0110009]

[3] F.Abe et al., CDF collaboration, Phys.Rev.Lett. 80 (1998) 2525 
[4] A.Aktas et al., H1 collaboration, Eur.Phys.J. C 33 (2004) 9

[5] H.Abramowicz et al., ZEUS collaboration, Phys.Lett.B 559 (2003) 153

[6] A.Aktas et al., H1 collaboration, Phys.Lett.B 561 (2003) 241, H1-prelim-05-164-PRC_Nov05

[7] J.Ferrando, ZEUS and H1 collaboration, contribution to EPS05, Lisboa, Portugal.

[8] H.Abramowicz et al., ZEUS collaboration, Phys.Lett.B 583 (2004) 41, H1-prelim-04-061, presented at DIS04 